\title{
Impact of Academic Stress on Depression Among Nursing Students: Testing for the Moderating Effect of Social Support
}

\author{
Jihye Lee ${ }^{1}$ and Moonhee Gang ${ }^{2}$ \\ ${ }^{1}$ Part-time lecturer, Department of Nursing, University of Ulsan, Ulsan, Korea \\ ${ }^{2}$ Professor, College of Nursing, Chungnam National University, Daejeon, Korea \\ jllulu@naver.com
}

\begin{abstract}
This study aimed to verify the moderating effect of social support in the relationship between academic stress and depression in nursing students. Therefore, 261 nursing students were recruited from October to November 2017. The collected data were analyzed using the SPSS/WIN 22.0 program with the Johnson-Neyman method in PROCESS. The effect of academic stress on depression was not statistically significant among nursing students with high social support. It was found that high social support can alleviate the effects of academic stress on depression in nursing students. To prevent depression among nursing students, it is necessary to continuously monitor the level of social support that nursing students were received.
\end{abstract}

Keywords: Student, Stress, Social, Support, Depression

\section{Introduction}

\subsection{Background}

Nursing students are under substantial amounts of stress due to the psychological pressure of having to pass a national examination and secure a job, the excessive amount of learning expected due to the rigorous curriculum, and the practice in various clinical situations [1]. In particular, the severity of academic stress was the highest among the other stresses experienced by nursing students [2], and it was higher than that experienced by dental and medical students [2][3]. Excessive academic stress can lead students to drop out from the program and may cause depression [4]. A study on medical graduate students found a significant correlation between depression and academic stress [5], and other studies reported that academic stress is a major factor affecting depression [3].

In fact, most nursing students were found to be in a mild depressive state [6] or to experience "mild" or "moderate" depression [2]. Studies on the relationship between stress, depression, and social support among Korean college students revealed that the level of their depressive symptoms and stress symptoms was lower because they perceived higher levels of social support from their parents [7], and the effect of stress on the depression of college students was found to be moderated by social support $[8,9]$. In addition, social support was found to have a buffering effect in the relationship between academic stress and depression in medical graduate schools [5]. Evidently, social support acts as a moderating variable in the

Article history:

Received (December 5, 2017), Review Result (February 12, 2018), Accepted (March 27, 2018) 
relationship between stress and depression. However, few studies have examined the moderating effect of social support on the relationship between academic stress and depression in nursing students, who have been reported to experience higher academic stress than students from other departments do. Thus, the purpose of this study was to investigate the moderating effects of social support on academic stress and depression in nursing students, and to help them prevent depression and adapt to the process of becoming a nurse.

\subsection{Purpose}

This study aimed to examine the moderating effects of social support on the relationship between academic stress and depression in nursing students.

\section{Methods}

\subsection{Study design}

This study employed a descriptive design to confirm the moderating effect of social support on the relationship between academic stress and depression in nursing students.

\subsection{Participants}

The subjects of this study were nursing students from 3 universities, who understood the purpose of the study and agreed to participate in the survey. The required sample size, determined by G-Power 3.1.9.2, was 222, to obtain an effect size of 0.15, at an alpha level of 0.05 and a power 0.95 , with 20 variables in the multiple regression analysis. Thus, the sample size of 240 exceeded the requirements of the power analysis.

\subsection{Instruments}

\subsubsection{Academic stress}

Academic stress was assessed using 10 study-related items from the 39 items assessing nursing students' stress developed by Yoo et al. [10]. Higher scores on these items indicate more severe stress levels. The reliability for the 10-item scale was Cronbach's alpha $=.88$ in the present study.

\subsubsection{Depression}

Depression was assessed using the integrated Korean version of the Center for Epidemiologic Studies Depression (CES-D) scale, which was developed for Korea by Chon et al. [11], based on the CES-D developed by Radloff [12]. Higher scores on this scale indicate more severe depression levels, and scores over 25 indicate probable depression. The reliability for this scale was Cronbach's alpha $=.93$ in the present study.

\subsubsection{Social support}

Social support was assessed using the Multidimensional Scale of Perceived Social Support (MSPSS) developed by Zimet et al. [13], and translated by Shin and Lee [14]. The MSPSS scale comprises 3 sub-scales (family support, friend support, and special support) and 12 items. Higher scores indicate a higher social support level. The reliability for this scale was Cronbach's alpha $=.77$ in the present study. 


\subsection{Data analysis}

The collected data were analyzed using SPSS/WIN 22.0. The general characteristics of the participants and the degree of academic stress, depression, and social support were analyzed using descriptive statistics. Further, the academic stress, depression, and social support scores were examined with reference to the general characteristics, using the t-test, an ANOVA, and the Scheffé test. The correlations between variables were analyzed using the Pearson's correlation coefficient. The moderating effect of social support on the relationship between academic stress and depression was examined using a hierarchical multiple regression analysis by centering the independent variables and moderating variables according to the method proposed by Baron and Kenny. This was followed by the analysis using the JohnsonNeyman method in PROCESS [15] to confirm the significance area and the comparison of the simple slope. The reliability of the tools used in the study was confirmed using the Cronbach's $\alpha$ coefficient.

\section{Results}

\subsection{Students' academic stress, depression, and social support}

In this study, the depression, academic stress, and social support scores of nursing students were $15.17 \pm 9.20,34.15 \pm 7.03$, and 46.09 \pm 11.37 , respectively. Depression and academic support showed a negative correlation $(\mathrm{r}=.28, \mathrm{p}<.001)$, while depression and social support showed an inverse correlation $(\mathrm{r}=.23, \mathrm{p}<.001)$. There was no statistically significant correlation between academic stress and social support.

\subsection{The moderating effect of social support on the relationship between students' academic stress and depression}

Academic stress had significant positive effects on depression $(\beta=0.29, \mathrm{p}<.001)$ while social support had significant negative effects on depression $(\beta=-0.29, \mathrm{p}<.001)$.

Table 1. Moderating effect of social support on the relationship between stress and depression $(\mathrm{N}=240)$

\begin{tabular}{|c|c|c|c|c|c|c|c|c|}
\hline Characters & B & SE & $\beta$ & $\mathrm{t}$ & $\mathrm{p}$ & VIF & $\begin{array}{l}\text { Durbin- } \\
\text { Watson }\end{array}$ & - \\
\hline Constant & 0.07 & .54 & & 0.13 & .899 & & \multirow{4}{*}{1.57} & \multirow{4}{*}{$\begin{array}{c}\mathrm{R}^{2}=17.2 \%, \\
\text { Adjusted } \\
\mathrm{R}^{2}=16.1 \%, \\
\mathrm{~F}=16.32, \\
\mathrm{p}<.001\end{array}$} \\
\hline Academic Stress (centered) & 0.38 & .08 & 0.29 & 4.90 & $<.001$ & 1.00 & & \\
\hline Social Support (centered) & -0.23 & .05 & -0.29 & -4.73 & $<.001$ & 1.06 & & \\
\hline $\begin{array}{c}\text { Academic Stress } \times \text { Social } \\
\text { Support }\end{array}$ & 0.00 & 0.01 & -0.19 & -3.05 & .003 & 1.06 & & \\
\hline
\end{tabular}

Additionally, the interaction between academic stress and social support was a significant predictor of depression $(\beta=-.19, \mathrm{p}=.003)$. The total explanatory power of academic stress, social support and the interaction between these variable was $16.1 \%$. As a result of exploring the significance areas using the John-Neyman method in PROCESS [17], the social support (centered values) were from 34.10. to 13.90. The effect of academic stress on depression was statistically significant only in areas with a social support score of less than 10.46, and it was not statistically significant in areas that exceeded this value. 
Figure 1 shows that the simple slopes identify the academic stress and depression values at the point where the social support values are -1 standard deviations $(\beta=-0.00, t=0.0918$, $\mathrm{p}<.001)$ and the mean values $(\beta=-0.00, \mathrm{t}=0.0918, \mathrm{p}<.001)$ are +1 standard deviations $(\beta=11.37$, $\mathrm{t}=1.75, \mathrm{p}=.082)$. In other words, high academic stress in those who receive low or moderate social support is associated with high depression, while academic stress is not significantly related to depression in highly-supported nursing students. The simple slope analysis showed that social support plays a moderating role in the relationship between academic stress and depression.

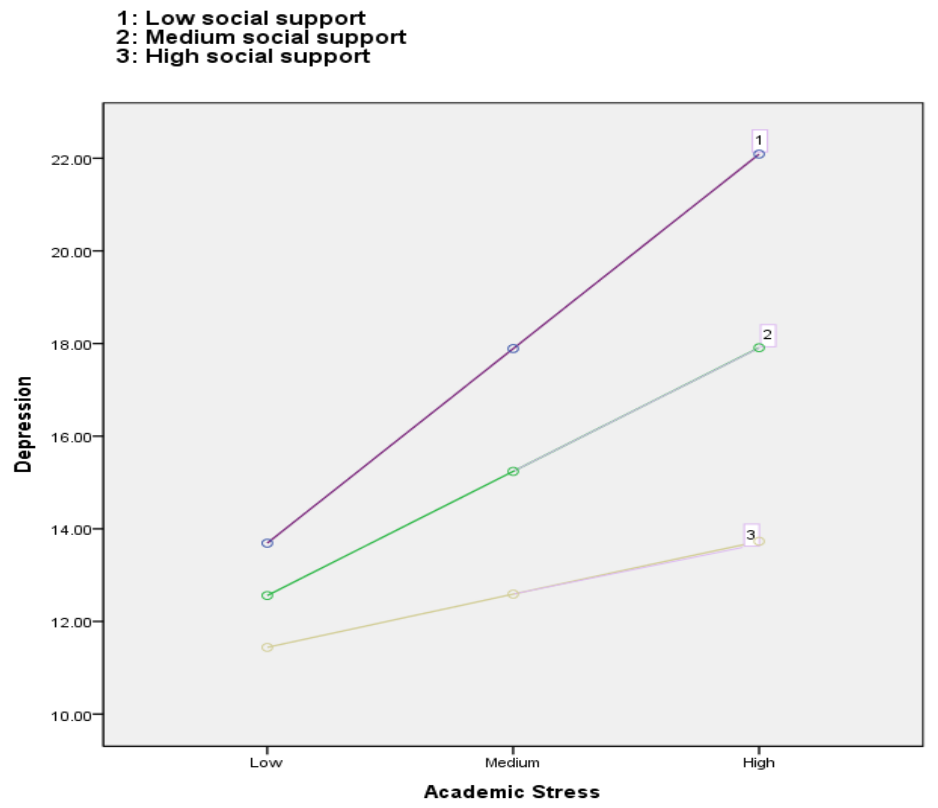

Figure 1. Interaction between academic stress and depression in nursing students: The moderating effect of social support

\section{Discussion}

Our findings confirmed that academic stress and social support are significant predictors of depression in nursing students, and that academic stress and social support had significant interaction effects on depression. Furthermore, social support was confirmed as a moderator in the relationship between academic stress and depression. These results are consistent with those of a study on university students, and with the results of previous studies examining the moderating effect of social support on the relationship between stress and depression [8][9]. Because a hierarchical regression analysis only reveals the presence or absence of moderating effects, in the present study, the significance area was examined using the John-Neyman method in PROCESS [15]. It was found that the influence of academic stress on depression was not significant among students with high social support. In addition, the simple slope revealed that high social support could alleviate depression. In other words, when the social support score was above 56.55, the effect of academic stress on depression was not significant. Thus, to prevent depression due to academic stress, it is possible to provide clear criteria for the sufficient level of social support required for nursing students. These findings also provide basic data for the development and evaluation of various intervention programs. However, as 
the present study was limited to a few nursing students and the survey cannot be considered at the time when it was performed, the generalizability of these findings is limited. Therefore, we propose that this study be replicated by using a study design that distinguishes between the test period and non-test period.

\section{Conclusion}

In this study, nursing students showed higher depression and academic stress than those from other department. Further, we found that social support has a moderating effect on the relationship between academic stress and depression in nursing students. Additionally, we analyzed this moderating effect with reference to the extent of social support, and confirmed that high social support can reduce the effect of academic stress on depression. Based on the present results, it is evident that we need to continuously review the social support level of nursing students and accordingly develop strategies to improve the same. In addition, we should continue to pay attention to the prevention of depression among nursing students.

\section{References}

[1] M.H. Jeong and M.A. Shin, "The relationship between premenstrual syndrome and menstrual attitudes of college students," The Journal of Korean Academic Society of Nursing Education, vol.12, no.1, pp.43-51, (2006)

[2] J.J. Kim, "Group art therapy effects on the nursing students for reduction of stress and depression," M.S. thesis, (Suwon): Aju University, (2013)

[3] J. Kang, Y.K. Ko, H. Lee, K. Kang, Y. Hur, and K. Lee, "Effects of self-esteem and academic stress on depression in Korean students in health care professions," Journal of Korean Academy of Psychiatric and Mental Health Nursing, vol.22, no.1, pp.56, (2013) DOI:10.12934/jkpmhn.2013.22.1.56

[4] S.H. Shin, "Influence from the academic stress by the achievement pressure of their parents and adjustment effect of self-differentiation," Journal of the Korea Academia-Industrial Cooperation Society, vol.15, no.11, pp.6756-6766, (2014) DOI: 10.5762/KAIS.2014.15.11.6756

[5] W.S. Lee, Y.J. Oh, and D.Y. Byun, "The academic stress, depression and social support of graduate medical school students: Testing the buffering effect of social support," Korean Journal of Social Welfare Research, vol.37, no.45, (2013)

[6] Y.J. Son, E.Y. Choi, and Y.A. Song, "The relationship between stress and depression in nursing college students," The Korean Journal of Stress Research, vol.18, no.4, pp.345-352, (2010)

[7] U.C. Kim, A. Tsuda, Y.S. Park, E.Y. Kim, and S. Horiuchi, "Comparative analysis of stress symptoms and management among Korean and Japanese university students: With a specific focus on the influence of social," The Korean Journal of the Human Development, vol.16, pp.139, (2009)

[8] Y.R. Park and E.H. Jang, "Impact of stress on depression among university students: testing for moderating effect of social support," Korean J. Adult Nurs, vol.25, no.5, pp.549, (2013) DOI:10.7475/kjan.2013.25.5.549

[9] X. Wang, L. Cai, J. Qian, J., and J. Peng, "Social support moderates stress effects on depression,” Int. J. Ment. Health Sys, vol.8, article no.41, (2014) DOI:10.1186/1752-4458-8-41

[10] J.S. Yoo, S.J. Chang, E.K. Choi, and J.W. Park, "Development of a stress scale for Korean nursing students," J. Korean Acad. Nurs, vol.38, no.3, pp.410-419, (2008) DOI: 10.4040/jkan.2008.38.3.410

[11] K.K. Chon, S.J. Choi, and B.C. Yang, "Integrated adaptation of CES-D in Korea," Korean Journal of Health Psychology, vol.22, no.3, pp.589, (2001)

[12] L.S. Radloff, "The CES-D scale: A self-report depression scale for research in the general population," Appl. Psychol. Meas, vol.1, pp.385, (1977)

[13] G.D. Zimet, N.W. Dahlem, S.G. Zimet, and G.K. Parley, "The multidimensional scale of perceived social support,” J. Pers. Assess, vol.52, no.1, pp.30-41, (1988) DOI: 10.1207/s15327752jpa5201_2 
Impact of Academic Stress on Depression Among Nursing Students: Testing for the Moderating Effect of Social Support

[14] J.S. Shin and Y.B. Lee, "The effects of social supports on psychosocial well-being of the unemployed," Korean Academy of Social Welfare, vol.37, pp.241-269, (1999)

[15] A.F. Hayes, "Editor, introduction to mediation, moderation, and conditional process analysis: A regressionbased approach," The Guilford Press, New York, (2013)

\section{Authors}

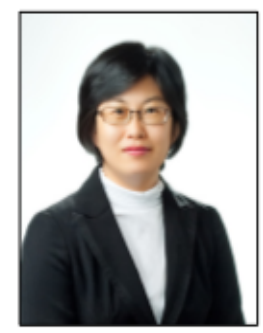

Moonhee Gang, Ph.D. is a professor of the College of Nursing at Chungnam National University. She is an RN and has the Ph.D. degree in Nursing Science from Chungnam National University. Her research interests are community mental health and dementia.

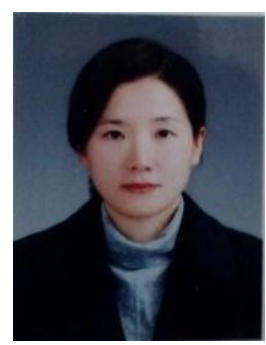

Jihye Lee, Ph.D. is a Part-time lecturer of Department of Nursing, University of Ulsan. She is an RN and has the Ph.D. degree in Nursing Science from Chungnam National University. Her research interests are communication, stress and dementia 